\title{
Penfigoide de membranas mucosas ocular: a propósito de un caso clínico
}

\author{
Arturo Grau Diez ${ }^{1}$, Rafael González Salazar ${ }^{1 *}$
}

Resumen - El Penfigoide de membranas mucosas (PMM), corresponde a un tipo de enfermedad ampollosa autoinmune, la que se caracteriza por presentar una alteración en la adhesión de las estructuras cutáneas. Su presentación ocular corresponde a una conjuntivitis crónica de difícil manejo que puede comprometer severamente la superficie ocular con formación de simbléfaron, acortamiento del fórnix, conjuntivalización y neovascularización corneal, conduciendo a la amaurosis total si no es tratado ni diagnosticado oportunamente. El diagnóstico por medio de biopsia de mucosa oral y conjuntival con estudio histológico y de inmunofluorescencia directa (IFD), aumenta considerablemente la sensibilidad y permite iniciar un tratamiento inmunosupresor sistémico disminuyendo la tasa de progresión de la enfermedad.

Palabras clave: penfigoide de membranas mucosas ocular; conjuntivitis cicatricial; inmunosupresión sistémica

Abstract - Mucous membrane pemphigoid (MMP), a type of autoimmune blistering disease, is characterized by an alteration in the adherence of skin structures. Ocular manifestations include chronic conjunctivitis that can severely compromise the ocular surface with symblepharon formation, shortening of the fórnix, conjunctivalization and corneal neovascularization, leading to total blindness if it is not diagnosed and treated promptly. The biopsy of oral and conjunctival mucosa for histological study and direct immunofluorescence (DIF) significantly increases sensitivity and allows the use of systemic immunosuppressive therapy to avoid the progression of the disease.

Keywords: ocular mucous membrane pemphigoid; cicatrizing conjunctivitis; systemic immunosuppression

Fecha de envío: 30 de octubre del 2015 - Fecha de aceptación: 16 de diciembre del 2015

\section{Caso Clínico}

Paciente de sexo femenino de 73 años con antecedentes de fibrilación auricular, hipertensión y artrosis en tratamiento. Con historia de dos años de evolución de tos crónica asociada a lesión faríngea, por lo que consulta en el Departamento de Otorrinolaringología y Odontología, diagnosticándose tos crónica secundaria a reflujo gastroesofágico y periodontitis avanzada crónica, respectivamente. Se realizó biopsia de tejido retrofaríngeo que fue descrito como un exudado fribrinopurulento y tejido granulatorio sin atipías citológicas.

Tras seis meses de tratamiento se diagnostican numerosas lesiones cutáneas ulceradas en la región genital las que evaluadas por el dermatólogo impresionan como liquen plano erosivo oral y genital. Se realiza una segunda biopsia documentada como lesión de aspecto benigno inflamatorio crónico nuevamente sin atipías celulares, no obstante sin diagnóstico certero. Tras la consulta reumatológica se efectúan estudios de laboratorio incluyendo factor reaumatoídeo, anticuerpos antinucleares, anticuerpos anticitoplasma de neutrófilos, pruebas treponémicas, función tiroidea, screening $\mathrm{VIH}$ y hematológico, con resultados dentro de parámetros normales.

Finalmente realiza una consulta oftalmológica por síntomas crónicos que incluyen prurito ocular, sensación de cuerpo extraño y ojo rojo bilateral. Al examen oftalmológico se observó fibrosis en ambos tarsos superiores, pérdida de pliegue semilunar bilateral y simbléfaron inferior en ojo derecho diagnosticándose conjuntivitis cicatricial crónica (Figura 1), por lo cual y en el contexto sistémico de la paciente, se realiza biopsia de mucosa conjuntival y oral (Figura 2 A y B) con estudio histológico y de IFD demostrando depósitos de IgG lineal y complemento (C3) en membrana basal epitelial (Figura 3), confirmando el diagnóstico de Penfigoide de membranas mucosas ocular (PMMO). Consecuentemente se inicia tratamiento

(1) Departamento de Oftalmología, Escuela Medicina, Pontificia Universidad Católica de Santiago de Chile

*Autor de Correspondencia: ragonzalez8@uc.cl 
Grau et al.

tópico con lubricantes sin conservantes y corticoides de superficie asociados a inmunosupresión sistémica con Dapsona 100mg día vía oral, con lo cual se logra controlar la sintomatología disminuyendo el proceso inflamatorio de superficie ocular y evitando la progresión de la cicatrización conjuntival ya establecida, recuperándose asimismo de las lesiones presentes en otras mucosas y mejorando notoriamente su calidad de vida.

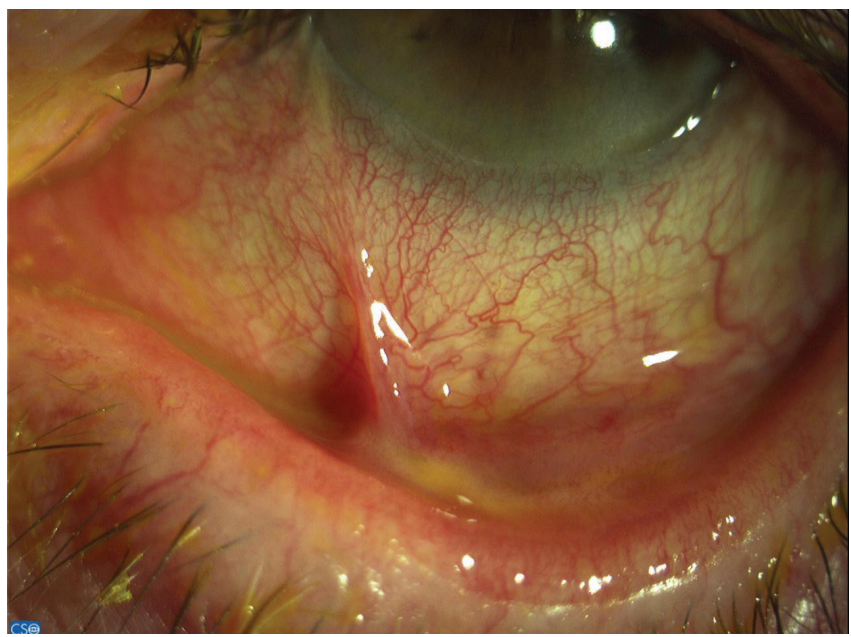

Figura 1: Simbléfaron. Cicatrización conjuntival tarsal bulbar progresiva

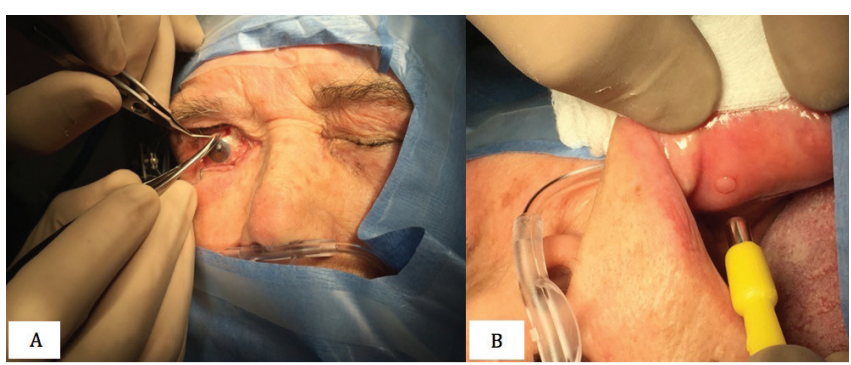

Figura 2: (A) Biopsia de conjunctiva bulbar superior. Se debe evitar la conjuntiva bulbar inferior y los fondos de saco por el riesgo de cicatrización anómala e infección. (B) Biopsia de mucosa bucal. Se aprecia la marca del punch dermatológico de $3 \mathrm{~mm}$.

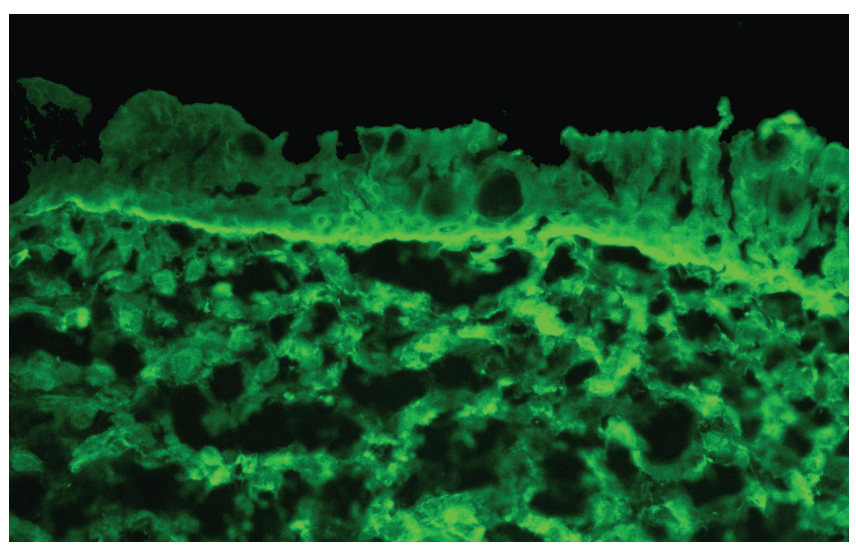

Figura 3: Inmunofluorescencia directa conjuntival

\section{Discusión}

El término "conjuntivitis cicatricial" engloba un conjunto de patologías que en su estadio final presentan cicatrización conjuntival progresiva, alterando severamente la superficie ocular con compromiso en todo nivel; párpados, fórnix conjuntival, córnea con formación de pannus, insuficiencia limbar y leucomas, todo lo cual puede conducir inevitablemente a la ceguera si no se realiza un manejo adecuado en etapas iniciales. Dentro del diagnóstico diferencial destacan infecciones por adenovirus, causticación ocular, Síndrome de Steven Johnson, queratoconjuntivitis atópica, Penfigoide de membranas mucosas ocular (PMMO), entre otras. Una historia clínica detallada en tanto a la forma de presentación, velocidad de progresión, noxa inicial, sumada a un exhaustivo examen oftalmológico y eventualmente la realización de una biopsia nos orientará al diagnóstico definitivo.

EL PMMO es un tipo de enfermedad ampollosa autoinmune, las que se caracterizan por presentar una alteración en la adhesión de las estructuras cutáneas, manifestándose en forma de ampollas y vesículas que tardíamente evolucionan a una cicatrización conjuntival patológica con neovascularización y opacificación corneal (Jordan et al., 1998). En un $80 \%$ de los casos presenta manifestaciones extraoculares comprometiendo mucosa oral, cavidad nasal, faringe y piel con $85 \%, 30 \%, 20 \%$ y $25 \%$, respectivamente (Schmidt \& Zillikens, 2013). Se estima una incidencia de 1.1 a 1.8 por millón de habitantes, siendo tres veces más frecuente en mujeres, con una edad promedio de 60- 70 años al momento del diagnóstico sin predilección por raza (Bernard et al., 1995).

Su fisiopatología conlleva a la formación de autoanticuerpos dirigidos contra proteínas estructurales de los complejos de unión (hemidesmosomas) entre las células epiteliales y la membrana basal, de ellas la integrina alfa 6 y beta 4 son las que con mayor frecuencia se asocian con compromiso ocular. La interacción antígeno-anticuerpo en la membrana basal produce una reacción inflamatoria en cascada con liberación de citoquinas, factor de necrosis tumoral e interleuquinas, ocasionando la separación del epitelio y su membrana basal con formación de ampollas subepiteliales tensas con contenido seroso, resultando una úlcera y posterior cicatriz residual en las mucosas afectadas (Xu et al., 2013). La gravedad de la enfermedad y su distribución es muy variable documentándose desde casos leves en los que sólo existe afectación de la mucosa oral en un $20 \%$, hasta aquellos extensos con importante compromiso de mucosa ocular, genital y laríngea.

La presentación oftalmológica del PMMO se basa en una conjuntivitis crónica inespecífica generalmente bilateral y asimétrica de progresión lenta. En su estadio inicial debuta con una fibrosis 
subepitelial que se detectará por la presencia de finas líneas pálidas subconjuntivales, siendo este hallazgo fundamental para un diagnóstico precoz. Al progresar la enfermedad se evidencia un acortamiento de los fondos de saco, pérdida del pliegue semilunar y la formación de simbléfaron (Figura 1), asimismo alteraciones palpebrales y corneales como triquiasis, distiquiasis, entropion cicatriciales por el acortamiento de los fondos de saco, la vascularización corneal, pannus, úlceras y leucomas corneales. En su grado final el PMMO presenta un compromiso terminal de la superficie ocular con hiperqueratosis de los epitelios conjuntival y corneal, amenazando la función visual, y puede alcanzar la amaurosis en un $27 \%$ de los casos (Espino-Barros \& Gonzalez-Gonzalez, 2010; Kourosh \& Yancey, 2011).

Si bien el diagnóstico en etapas iniciales requiere un alto índice de sospecha en casos de conjuntivitis crónicas que no respondan a tratamiento convencional, su confirmación diagnóstica se realiza en base a la clínica, exploración oftalmológica y el estudio histopatológico y por medio de IFD de muestras obtenidas en la mucosa oral y conjuntival. La muestra conjuntival aislada tiene un rendimiento de un $60 \%-80 \%$ dependiendo de la actividad de la enfermedad, por su parte el estudio de mucosa oral es positivo en un $70 \%$ de los casos aun en ausencia de lesiones bucales, sin embargo, la ejecución del estudio de ambos tejidos aumenta el rendimiento considerablemente (Thorne et al., 2004; Goldich et al., 2015). Para la realización del estudio mediante biopsia, se recomienda obtener tejido de la conjuntiva bulbar superior debido a que el párpado cubre esa área y mejora la cicatrización (Figura 2a). Se debe evitar la conjuntiva bulbar inferior y los fondos de saco por el riesgo de cicatrización anómala.

\section{Biopsia conjuntival y de mucosa oral}

Para la toma de muestra de mucosa conjuntival, se instila anestésico tópico utilizando un blefarostato o simplemente la separación de párpados manualmente con uso de tijera y pinzas de conjuntiva para lograr una muestra de 2-4 mm. Durante el procedimiento se realiza además la obtención de mucosa bucal independientemente a que el paciente presente compromiso oral. Debe inyectarse el anestésico evitando el área del conducto parotídeo, que se encuentra cercano al segundo molar y para la recolección de tejido se utiliza un punch dermatológico de $4 \mathrm{~mm}$, tijera y pinza, y sin la necesidad de sutura posterior (Figura 2b). El tratamiento postoperatorio consiste en colirio de antibiótico y corticoides por 4 semanas con la finalidad de disminuir el riesgo de cicatrización patológica, pudiendo utilizarse enjuagues bucales antisépticos por algunos días (Grau et al., 2013). Es importante enfatizar que el traslado de la muestra para estudio mediante IFD se realiza en solución de transporte de Michel, la cual mantiene los complejos inmunológicos en su sitio histológico permitiendo analizar su patrón de depósito. Si bien un resultado negativo no descarta la enfermedad, la presencia de depósitos lineales de lgG y/o complemento en la membrana basal del epitelio son diagnósticos de PMMO (Figura 3) y debe complementarse con el estudio histológico, que básicamente ayuda a descartar cuadros neoplásicos, atópicos y granulomatosos (Hervás Ontiveros \& Salom, 2014).

El abordaje terapéutico del PMMO tiene como ejes principales el control de la inflamación, fibrosis y compromiso corneal con optimización de la función visual. El manejo general de la blefaritis, ojo seco, queratitis filamentosa, disminución de la queratinización de la superficie y de los defectos epiteliales recurrentes van de la mano con la terapia inmunosupresora. Se establece una terapia sistémica escalonada dependiendo del grado de severidad de la enfermedad (Figura 4), teniendo como opciones para cuadros leves la Dapsona y Sulfapiridina, en casos moderados Azatioprina y Micofenolato y en aquellos severos o que no responden a tratamiento Ciclofosfamida, sopesando riesgos en cuanto a reacciones adversas y beneficios para cada paciente. Dicho plan inmunosupresor debe ser indicado en conjunto con médico reumatólogo (Saw \& Dart, 2008; Saw et al., 2008). En PMMO refractario a tratamiento se han realizado estudios recientes con terapia biológica tales como Inmnuglobulinas intravenosas, Etanercept, Infliximab, Daclizumab o Rituximab, este último con buena respuesta y bajas reacciones adversas (Le Roux-Villet et al., 2011; Rubsam et al., 2015).

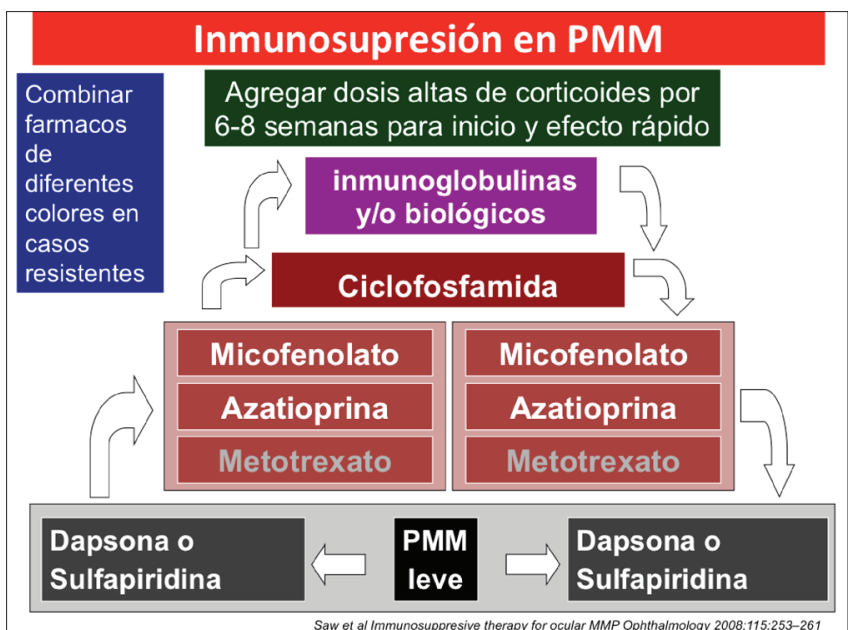

Figura 4: Esquema de manejo terapéutico del PMMO.

Hasta un 30\% requiere algún tipo de reparación quirúrgica de los defectos cicatriciales, para ello es fundamental realizar una terapia inmunosupresora previa y posteriormente a la cirugía con el objeto de evitar la reactivación de la enfermedad y la aparición de un nuevo proceso cicatricial, logrando en la mayoría de los casos resultados finales satisfactorios (Gibbons et al., 2015). Lamentablemente y a pesar del diagnóstico e intervenciones terapéuticas precoces, 
Grau et al.

algunos pacientes son refractarios al tratamiento. Radford y cols, documentan que hasta un $26 \%$ de los ojos presentan progresión a pesar de la inmunosupresión. Por otro lado, el control de la enfermedad y su tratamiento se ve dificultado por el hecho de que puede existir progresión sin evidencia clínica de inflamación. Estos datos exhiben no solo el reto terapéutico que representa la enfermedad per se, sino también su pronóstico reservado (Radford et al., 2012).

En vista de lo expuesto, podemos concluir que el PMMO corresponde a una enfermedad multisistémica de manejo interdisciplinario que requiere una alto índice de sospecha clínica para no retrasar el diagnóstico y realizar un tratamiento oportuno. Su confirmación diagnóstica se realiza en base a la biopsia de mucosa conjuntival y bucal, lo que permite el análisis histopatológico y de IFD. Un tratamiento inmunosupresor sistémico escalonado y controlado es la base para disminuir la progresión de la enfermedad, evitando el riesgo de ceguera y la frecuente morbilidad asociada.

\section{Referencias}

Bernard P, Vaillant L, Labeille B, Bedane C, Arbeille B, Denoeux JP, Lorette G, Bonnetblanc JM \& Prost C. (1995). Incidence and distribution of subepidermal autoimmune bullous skin diseases in three French regions. Bullous Diseases French Study Group. Arch Dermatol 131, 48-52.

Espino-Barros PA \& Gonzalez-Gonzalez LA. (2010). Presentación clínica y manejo del penfigoide cicatricial en México. Rev Mex Oftalmol 84, 10-18.

Gibbons A, Johnson TE, Wester ST, Gonzalez AE, Farias CC, Betancurt C, Amescua G \& Perez VL. (2015). Management of patients with confirmed and presumed mucous membrane pemphigoid undergoing entropion repair. Am J Ophthalmol 159, 846-852.

Goldich Y, Ziai S, Artornsombudh P, Avni-Zauberman N, Elbaz U, Rootman DS \& Chan CC. (2015). Characteristics of patients with ocular cicatricial pemphigoid referred to major tertiary hospital. Can J Ophthalmol 50, 137-142.
Grau AE, Setterfield J \& Saw VPJ. (2013). Br J Ophthalmol 97, 530-531. Hervás Ontiveros A \& Salom D. (2014). Arch Soc Esp Oftalmol 89, 447-449.

Jordan E, Scott BS \& Razzaque AA. (1998). The blistering diseases. Med Clin North Am 82, 1239-1283.

Kourosh AS \& Yancey KB. (2011). Pathogenesis of mucous membrane pemphigoid. Dermatol Clin 29, 479-484.

Le Roux-Villet C, Prost-Squarcioni C, Alexandre M, Caux F, Pascal F, Doan S, Brette MD, Soued I, Gabison E, Aucouturier F, Letestu R, Laroche $L$ \& Bachelez H. (2011). Rituximab for patients with refractory mucous membrane pemphigoid. Arch Dermatol 147, 843-849.

Radford CF, Rauz S, Williams GP, Saw VP \& Dart JK. (2012). Incidence, presenting features, and diagnosis of cicatrising conjunctivitis in the United Kingdom. Eye (Lond) 26, 1199-1208.

Rubsam A, Stefaniak R, Worm M \& Pleyer U. (2015). Rituximab preserves vision in ocular mucous membrane pemphigoid. Expert Opin Biol Ther 15, 927-933.

Saw VP \& Dart JK. (2008). Ocular mucous membrane pemphigoid: diagnosis and management strategies. Ocul Surf 6, 128-142.

Saw VPJ, Dart JKG, Rauz S, Ramsay A, Bunce C, Xing W, Maddison PG \& Phillips M. (2008). Immunosuppressive Therapy for Ocular Mucous Membrane Pemphigoid. Ophthalmology 115, 253-261.e251.

Schmidt E \& Zillikens D. (2013). Pemphigoid diseases. Lancet 381, 320-332.

Thorne JE, Anhalt GJ \& Jabs DA. (2004). Mucous membrane pemphigoid and pseudopemphigoid. Ophthalmology 111, 45-52.

Xu HH, Werth VP, Parisi E \& Sollecito TP. (2013). Mucous membrane pemphigoid. Dent Clin North Am 57, 611-630. 